\title{
Generalized Demodulation Transform for Bearing Fault Diagnosis Under Nonstationary Conditions and Gear Noise Interferences
}

\author{
Dezun Zhao ${ }^{1}$, Jianyong $\mathrm{Li}^{2,3}$, Weidong Cheng ${ }^{2^{*}}$ and Zhiyang $\mathrm{He}^{2}$
}

\begin{abstract}
It is a challenging issue to detect bearing fault under nonstationary conditions and gear noise interferences. Meanwhile, the application of the traditional methods is limited by their deficiencies in the aspect of computational accuracy and efficiency, or dependence on the tachometer. Hence, a new fault diagnosis strategy is proposed to remove gear interferences and spectrum smearing phenomenon without the tachometer and angular resampling technique. In this method, the instantaneous dominant meshing multiple (IDMM) is firstly extracted from the time-frequency representation (TFR) of the raw signal, which can be used to calculate the phase functions (PF) and the frequency points (FP). Next, the resonance frequency band excited by the faulty bearing is obtained by the band-pass filter. Furthermore, based on the PFs, the generalized demodulation transform (GDT) is applied to the envelope of the filtered signal. Finally, the target bearing is diagnosed by matching the peaks in the spectra of demodulated signals with the theoretical FPs. The analysis results of simulated and experimental signal demonstrate that the proposed method is an effective and reliable tool for bearing fault diagnosis without the tachometer and the angular resampling.
\end{abstract}

Keywords: Bearing fault diagnosis, Generalized demodulation transform, Nonstationary conditions, Gear noise

\section{Introduction}

Bearing failure is one of the most common causes for rotating machinery breakdowns and accidents, and condition monitoring and fault detection of the bearing has been a subject of extensive research in recent years [1-3]. With efforts of the researchers, bearing fault information can be extracted from vibration, sound, current, acoustic emission and temperature signals. In particular, vibration signal analysis techniques, such as the time-domain statistics [4], the spectrum [5] and the time-frequency analysis [6], are the preferred approaches due to their satisfactory performance.

However, the bearing vibration signals are inevitably contaminated by background noise especially the strong interferences from gearbox. In industry, the bearing is often installed nearby the gearbox, even in the gearbox.

\footnotetext{
*Correspondence: wdcheng@bjtu.edu.cn

${ }^{2}$ School of Mechanical Electronic and Control Engineering, Beijing

Jiaotong University, Beijing 100044, China

Full list of author information is available at the end of the article
}

Hence, the gear meshing vibration always makes great difficulty to extract bearing fault feature. As shown in Figure 1, there are many interference peaks such as the drive gear rotating frequency and the driven gear rotating frequency in the envelope spectrum of faulty bearing signal with gearbox interferences. In addition, the peak corresponding to fault characteristic frequency (FCF) of the target bearing is not prominent. In this case, bearing fault cannot be detected easily.

Another important influence for bearing fault diagnosis is nonstationary conditions, which is common in real applications $[7,8]$. The variable rotational speed will result in the time-varying FCF. In this case, the traditional signal analysis methods based on the assumption of stationary conditions are no longer applicable. Figure 2 shows the envelope spectrum of faulty bearing signal under nonstationary condition. The figure reveals the obvious frequency smearing phenomenon, and does not reveal the FCF. Therefore, it is a significant issue to detect the interested information from faulty bearing vibration 


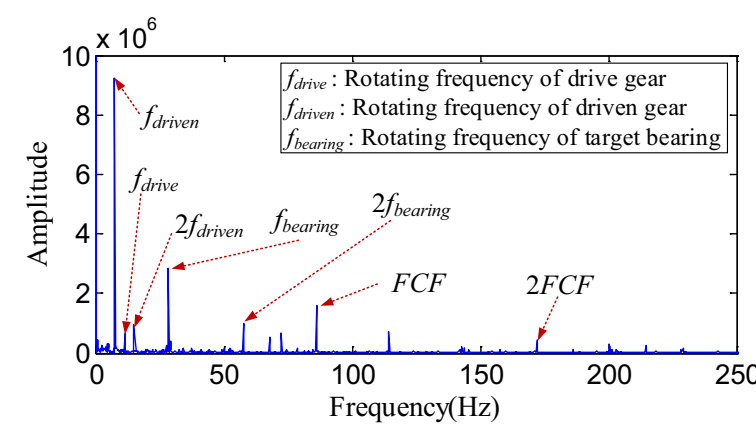

Figure 1 Envelope spectrum of faulty bearing signal with gearbox interference

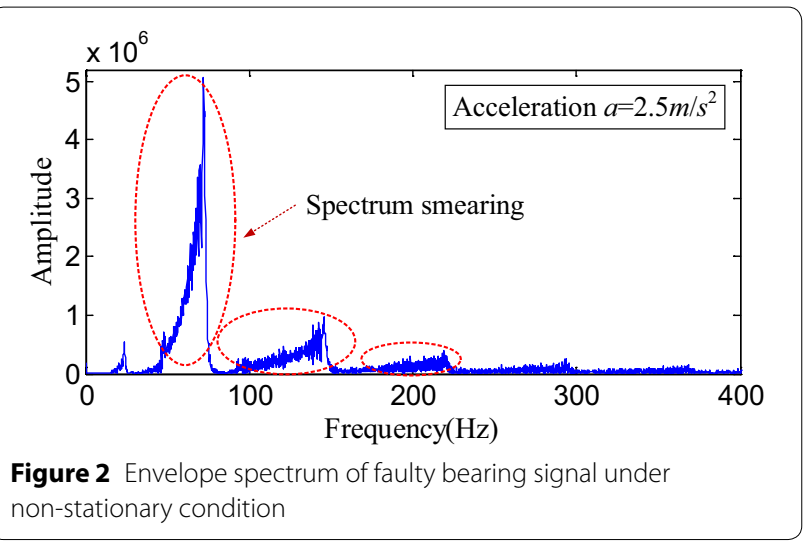

signal under nonstationary conditions and gear noise interference.

To tackle this issue, the most common methods are combining the computed order analysis (COA) and the gear noise elimination-based algorithms. Borghesani et al. [9] proposed an algorithm which includes eliminating influences of speed fluctuation with the angular resampling, removing gear interferences by the denoising method, applying the reverse order tracking and the angular resampling to the denoised signal, and detecting fault by the envelope analysis. This method is complicated and critically depends on the tachometer which is not always available. Focused on the above defects, Wang et al. [10] proposed an adaptive noise cancellation-based method. In this method, the IDMM trend is extracted from the TFR of the raw signal as a reference to remove the gear noise, then with the help of instantaneous fault characteristic frequency extracted from the envelope TFR of the denoised signal, the COA is applied to diagnose fault bearing. In addition, a bearing fault diagnosis method based on the empirical mode decomposition and the COA is proposed in Ref. [11]. Compared with the method proposed by Borghesani et al., the latter two methods can effectively remove the limitation of the tachometer. However, all the above methods rely on the COA.

The COA is an effective method to transform the nonstationary signal (in time domain) into the stationary one (in angle domain) for eliminating the influences of speed fluctuation with help of rotating speed or angular position measured by additional sensor [12-14], even the instantaneous rotating frequency can be extracted or estimated $[15,16]$, the deficiencies in aspect of computational accuracy and efficiency cannot be ignored. Saavedra et al. [17] pointed out that the computational errors are caused by calculation of resampled times and estimation of resampled amplitude. Cheng et al. [18] pointed out that the deformation of the envelope signal causes a variation in the angular interval between the peaks of the impulses, which will directly affect the accuracy of envelope analysis results. Furthermore, lots of equations calculation for determining the angular positions is timeconsuming. Lastly, bearing fault type is determined by the ratio between the fault characteristic order and the rational frequency order, which isn't obvious in some case. The unobvious rational frequency order brings challenges to locate fault.

The GDT is developed by Olhede et al. [19] for speechtype signal processing. This method can transform an arbitrary time-varying frequency ridge into a line paralleling to the time axis, and make it possible to separate any interested time-frequency ridge into a mono-component from other components by conventional filters. For separating the overlapped multi-components, an improved GDT is proposed in Ref. [20]. In this improved method, a varying window function is used to improve the time-frequency concentration of the generalized short time Fourier transform. Due to the characteristics of the GDT, some researchers applied it or its improved versions to process the nonstationary signals, especially for processing amplitude modulation (AM) -frequency modulation (FM) signal in recent years [21, 22]. A joint envelope and frequency order spectrum analysis method based on iterative GDT is proposed by Feng et al. [23] for diagnosing planetary gearbox under nonstationary conditions, where the iterative GDT can decompose all the constituent components into mono-components of constant frequency.

According to the above analysis, a satisfactory bearing fault diagnosis strategy should be able to eliminate the influences of gear noise and rotating speed fluctuation without the COA, the tachometer and the complicated denoising algorithm. As such, based on the GDT, the bearing high frequency resonance phenomenon and the gear meshing characteristic, a new bearing fault diagnosis method under nonstationary conditions and gear noise interferences is developed. In the method, the prominent 
time-frequency ridge named IDMM is firstly extracted from the TFR of raw signal, and the extracted IDMM can be used to calculate the PFs and FPs. Secondly, the bandpass filter is used to separate faulty bearing vibration and gearing meshing vibration due to their different distributed levels in the TFR, and based on the AM-FM features of bearing vibration signal $[24,25]$ the envelope of the filtered signal including FCF is obtained by the Hilbert transform. Lastly, the GDT is applied to the envelope signal for transforming the time-varying FCF and its harmonics into straight lines paralleling to the time axis, in this case, the peaks represented FCF and its harmonics can be easily captured in envelope spectrum.

The rest of this paper is structured as follows. In Section 2, theoretical basis of the proposed method is presented. Simulated signal is constructed for validating the proposed method in Section 3. In Section 4, the proposed method is further examined by different faulty bearing signals. The conclusions are presented in Section 5.

\section{Theoretical Background}

\subsection{Gear Meshing Vibration Characteristic}

As shown in the previous researches, gears meshing vibration is generally classified as narrowband periodic signal [26] and the meshing frequency trendline is prominent in the TFR of vibration signal even the gear has no faults, where the dominant meshing harmonic $f_{m d}$ is given by

$$
f_{m d}=c_{d} \times f_{m}=c_{d} \times \frac{z_{i} n_{i}}{60},
$$

where $c_{d}$ is the serial number of the dominant meshing harmonic, whose value is 2 or 3 in the most general case; $f_{m}$ is the gear meshing frequency; $z_{i}$ is the number of gear teeth, whose rotational speed is represented by $n_{i}$ and the unit is $\mathrm{r} / \mathrm{min}$.

In general, vibration signals generated from a gearbox or gearbox-based rotor-bearing system are dominated by gear meshing vibration $[27,28]$. Figure 3 shows the TFR and spectrum of measured signal including faulty bearing signal and gear meshing vibration, respectively. In Figure 3, the IDMM (around $610 \mathrm{~Hz}$ ) has obvious amplitude superiority compared with the vibration excited by the faulty bearing (from $6000 \mathrm{~Hz}$ to $10000 \mathrm{~Hz}$ ).

Hence, the IDMM can be easily extracted from the TFR of the raw signal. In this paper, the Short-time Fourier transform (STFT) is applied to calculate the TFR of the raw signal. The STFT is represented as follows:

$$
S_{x}(t, \xi)=\int_{-\infty}^{+\infty} x(\tau) g(\tau-t) e^{-i \xi \tau} \mathrm{d} \tau,
$$

where $t$ is the time shift, $\zeta$ denotes the frequency, $g(\tau)$ is the window function. Base on Eq. (2), the set of IDMM can be calculated by
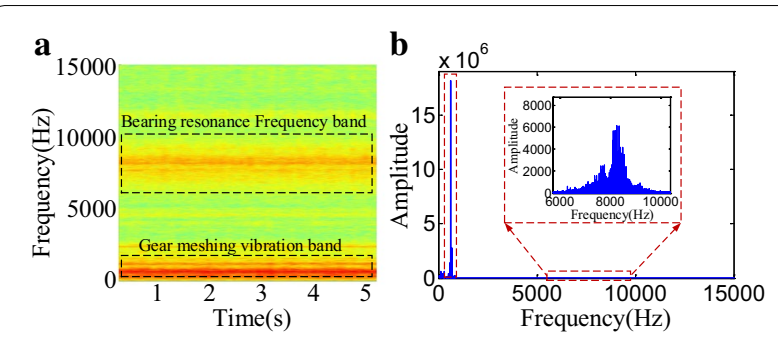

Figure 3 Introduction of IDMM: a TFR; $\mathbf{b}$ Spectrum

$$
f_{m d}=\operatorname{argmax}\left(S_{i}\right),
$$

where $S_{i}$ is the instantaneous spectrum; $i(i=1,2, \ldots, n)$ represents the sequence number of instantaneous spectrum and it is determined by the length of the Gaussian window function; $\operatorname{argmax}(\cdot)$ is peak search algorithm.

The IDMM function can be given by

$$
f_{m d}(t)=f_{m d}\left(t, a_{1}, a_{2}, \ldots, a_{m}\right),
$$

where $a_{m}$ is undetermined coefficient obtained by tendency of the IDMM tend.

\subsection{High Frequency Resonance and AM-FM Characteristic of Faulty Bearing Signal}

When a rolling element bearing has a localized fault, the contact of the defect and its mating surface will excites a transient impulse response with a short duration and an approximately exponential damping rate. The repetition frequency of the impulse, i.e., the FCF has constant ratio with rotational frequency. In addition, the fault occurred on different parts have different ratios, which can be calculated by the following equations [12]:

$$
\begin{aligned}
& F_{o}=\frac{f_{o}}{f_{r}}=\frac{n}{2}\left(1-\frac{d}{D} \cos \alpha\right), \\
& F_{i}=\frac{f_{i}}{f_{r}}=\frac{n}{2}\left(1+\frac{d}{D} \cos \alpha\right), \\
& F_{b}=\frac{f_{b}}{f_{r}}=\frac{D}{2 d}\left[1-\left(\frac{d}{D}\right)^{2} \cos ^{2} \alpha\right],
\end{aligned}
$$

where $F_{o}, F_{i}$ and $F_{b}$ are fault characteristic coefficients (FCC) of the out race, inner race and ball, respectively; $f_{o}, f_{i}$ and $f_{b}$ are FCFs of the out race, inner race and ball, respectively; $f_{r}$ is the rotational frequency of the target bearing; $n$ is the number of the ball; $d$ and $D$ represent the diameter of the ball and the pitch, respectively; $\alpha$ is the angle of the load from the radial plane.

Meanwhile, the localized fault on a bearing will excites a mechanical structural resonance in operating condition, and the FCF of the bearing will be 
modulated by the resonance band. Therefore, faulty bearing vibration signals often exhibit AM-FM phenomenon. In general, the resonance band excited by bearing fault is located at a frequency band far from the gear meshing vibration as shown in Figure 3. The region of gear meshing vibration is around from $0 \mathrm{~Hz}$ to $700 \mathrm{~Hz}$, and the faulty bearing vibration has high level from $5500 \mathrm{~Hz}$ to $10000 \mathrm{~Hz}$. As such, high frequency resonance band that including bearing fault information can be separated by the band-pass filter.

The high frequency resonance band can be represented by [24]

$$
x(t)=\sum_{m=1}^{M} A_{m}(t) \cos \left[2 \pi \varphi_{m}(t)\right],
$$

where $A_{m}$ is the amplitude of the $m$ th impulse, $m$ is the number of the impulses, $\varphi(t)$ is the instantaneous phase of the AM-FM component. The analytical function of the $x(t)$ is given by [29]

$$
z[x(t)]=x(t)+j H[x(t)]=\sum_{m=1}^{M} A_{m}(t) e^{j \varphi_{m}(t)},
$$

where $H[\cdot]$ represents the Hilbert transform; $\varphi_{m}(t)$ is the $m$ th harmonics of the characteristic phase, and the envelope amplitude is given as

$$
\hat{x}(t)=\sum_{m=1}^{M} A_{m}(t)=\sqrt{x^{2}(t)+\{H[x(t)]\}^{2}} .
$$

Based on the above analysis, it can be concluded that the FCF and its harmonics have amplitude advantage in the envelope of high frequency resonance signal. Under time-varying speed conditions, the time-frequency ridges in envelope TFR will change with time, which is the main reason for the spectrum smearing. In traditional methods, angular resampling is used to transform the time-varying ridges into lines paralleling to the angular axis, and the fault characteristic order and rotational frequency order can be captured in the envelope order spectrum, then the bearing fault type is determined. Inevitably, if the rotational frequency order is not obvious, the bearing fault cannot be determined. Most importantly, other irrelevant components are also transformed which will bring interferences for detecting the fault characteristic order and its harmonics. Inspired by angular resampling technique, if an algorithm can be used to reset the interested timevarying frequency ridge only, and new parameter is used to quantize the FCF and define fault type, the faulty bearing can be diagnosed.

\subsection{Generalized Demodulation Transform}

The GDT is motivated by the modulation or frequency shift property of the Fourier transform, and its essence is the generalized Fourier transform. For an arbitrary signal $x(t)$, its Fourier transform can be expressed as

$$
X_{G}(f)=\int_{-\infty}^{+\infty} x(t) e^{-2 j \pi\left[f \cdot t+s_{0}(t)\right]} \mathrm{d} t,
$$

where $s_{0}(t)$ is a real-valued function depending on the time only, which represents the PF of the signal. This is the same as applying the standard Fourier transform to $x(t) e^{-2 \pi j s_{0}(t)}$.

Based on Eq. (11), it can be found that if $X_{G}(f) \equiv \delta\left(f-f_{0}\right)$, $x(t)$ can be written as

$$
x_{d}(t)=e^{2 \pi j\left[f_{0} \cdot t+s_{0}(t)\right]} .
$$

Equation (12) shows that in a modulated signal, an interested time-varying frequency ridge can be transformed into a constant one, as long as its PF $s_{0}(t)$ satisfies

$$
f(t)=f_{0}+\mathrm{d} s_{0}(t) / \mathrm{d} t,
$$

where $f(t)$ is equation of the interested time-varying frequency ridge; $f_{0}$ is a constant.

Namely, if the function of the interested time-frequency curve is calculated, the time-varying frequency ridge can be transformed into linear path paralleling to the time axis.

The steps of the GDT are briefly summarized as follows:

1. Obtain the equation of the target time-frequency trajectory;

2. Calculate its PF based on Eq. (13);

3. Based on the PF, the GDT is applied to the analytic signal $x(t)$, and a new signal $d(t)=x(t) e^{-2 \pi j s_{0}(t)}$ is obtained;

4. An analytic signal $z(t)=d(t)+j H(t)$ is obtained to avoid negative frequencies, and it is the final demodulated result.

An arbitrary mono-component signal $x(t)=$ $\sin \left[(25 t-31)^{2} t+1257 t\right]$ is constructed to verify the GDT, whose instantaneous frequency function $f(t)=298 t^{2}-493 t+353$ is calculated.

Based on the instantaneous frequency $f(t)$, its PF $s_{0}(t)=99 t^{3}-246.5 t^{2}$ is calculated. The TFRs of the constructed signal and its demodulated signal are shown in Figure 4(a) and (b), respectively. As shown in Figure 4(a) and (b), the time-varying frequency ridge is mapped into a line paralleling to the time axis, and the frequency concentration point is equal to frequency starting point of the original time-frequency trajectory. The TFRs of original signal and demodulated 

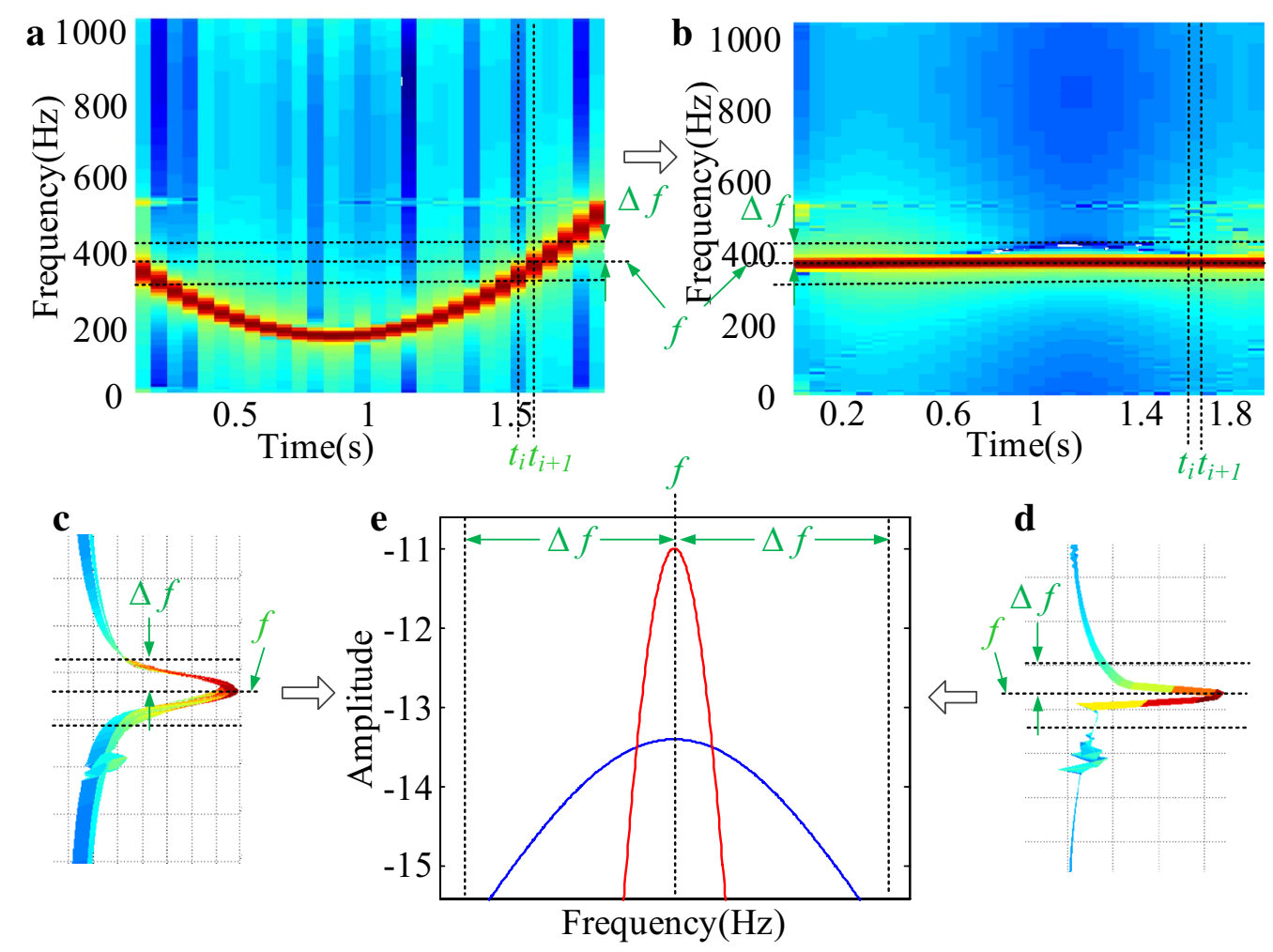

Figure 4 Illustration of GDT: a TFR of raw signal; bTFR of demodulated signal; c Partial enlarged drawing of TFR of raw signal; d Partial enlarged drawing of TFR of demodulated signal; e Amplitude comparison

signal are segmented with a short window $[f-\Delta f$, $f+\Delta f$ from $t_{i}$ to $t_{i+1}$, and the results are presented in Figure $4(\mathrm{c})$ and $(\mathrm{d})$, respectively. The section diagram of the above two segments is displayed in Figure 4(e) in which the time-frequency ridge of the demodulated signal is much more concentrated compared with the ridge in the constructed signal.

Based on the above analysis results, two important properties of the GDT can be summarized as follows:

1. A time-varying component can be converted into a line paralleling to the time axis, and the energy of the original signal would be concentrated on the constant frequency, which is equal to the frequency starting point of the original time-frequency path.

2. The energy concentration degree of the time-frequency trajectory in the TFR of the demodulated signal is improved.

\subsection{Overall Framework of the Proposed Method}

Based on the above methodology, a bearing fault diagnosis method is proposed. The flowchart of the proposed method is shown in Figure 5. Once the vibration signal of faulty bearing is obtained, the main processing steps are conducted as follows.

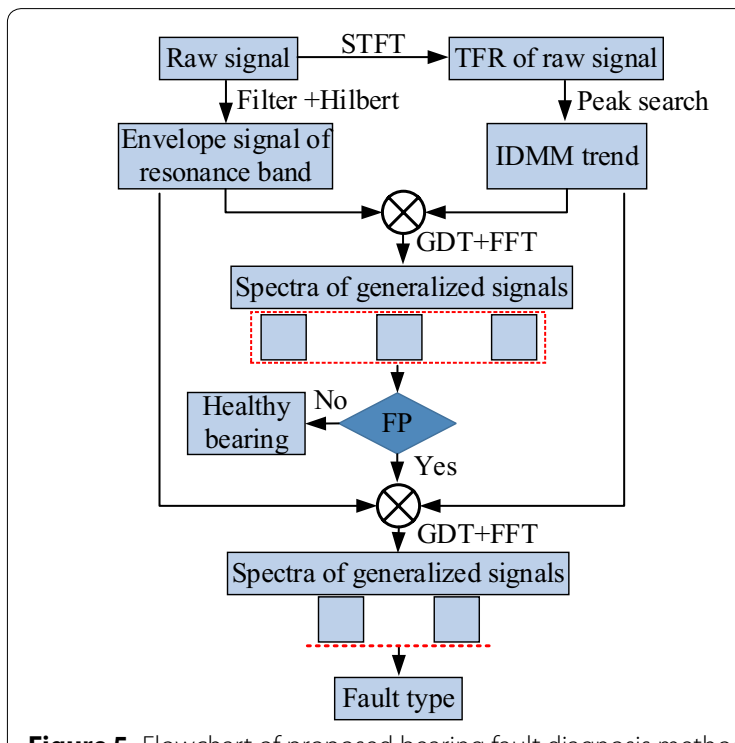

Figure 5 Flowchart of proposed bearing fault diagnosis method 
1. Calculated the TFR of the raw signal, from which the IDMM is extracted by the peak search algorithm;

2. Envelope of resonance frequency signal is obtained via band-pass filter and Hilbert transform, where the filtering parameters are estimated from the TFR of the raw signal;

3. The PFs and FPs corresponding to the different fault types are calculated by the IDMM function and mechanical structure parameters;

4. The GDT is applied to envelope signal with different $\mathrm{PFs}$, and then the spectra of demodulated signals are obtained by the fast Fourier transform (FFT);

5. Fault diagnosis by locating the peak in the spectra with the FPs;

6. The envelope signal is demodulated again with PFs for further determining the fault type of the target bearing, where the PFs correspond to the second and third harmonics of the captured peak in Step (5).

\section{Simulation Verifications}

A simulated signal including the faulty bearing signal, gear interference and white Gaussian noise is constructed to test the proposed method in this section. The simulated model of faulty bearing signal under nonstationary conditions is presented as follows [30]:

$$
\begin{aligned}
x_{b}(t)= & \sum_{i=1}^{I} A_{i} e^{-\beta\left(t-t_{i}-\sum_{j=1}^{i} \tau_{j}\right)} \sin \left[\omega\left(t-t_{i}-\sum_{j=1}^{i} \tau_{j}\right)\right] \\
& \cdot u\left(t-t_{i}-\sum_{j=1}^{i} \tau_{j}\right),
\end{aligned}
$$

where $I$ is number of the fault impulses; $A_{i}, A_{i}=t_{i}+1.5$, is the amplitude of the $i$ th fault impulse; $\beta$ is the structural damping characteristic, $\omega$ denotes resonance frequency excited by local fault; $\tau_{i}$ denotes the coefficient to calculate slippage time which varies from 0.01 to $0.02 ; \mu(t)$ is a unit step function; $t_{i}$ represents the occurrence time of the $i$ th impulse calculated as

$$
t_{i}=\frac{i}{F \times f_{b}(t)},
$$

where $f_{b}(t), f_{b}(t)=2.5 t+15$, is the rotational frequency function; $F$ is the FCC.

In general, vibration signals of a healthy gearbox are dominated by gear meshing, whose simulated model under nonstationary conditions can be represented as

$$
x_{g}(t)=\sum_{j}^{J} X_{j}(t) \cos \left[2 \pi j z F_{g}(t)\right],
$$

where $j(j=1,2, \ldots, J)$ is the meshing multiple number and $X_{j}(t)$ is the amplitude of $j$ th harmonic frequency. $z$ is the tooth number. $F_{g}(t)$ is integral function of the gear rotational frequency $f_{g}(t), c$ is the ratio of gear rotational frequency to bearing, namely $f_{g}(t)=c f_{b}(t)$. The amplitudes of gear meshing frequency $X_{1}=0.4$, and amplitudes of its 2nd and 3rd multiples are set as 4 and 0.3 , which means that the 2nd multiple is the dominant meshing multiple. The other parameters in the models are listed in Table 1.

Based on Eqs. (14)-(16), the simulated signal is given by

$$
x(t)=x_{b}(t)+x_{g}(t)+n(t),
$$

where $n(t)$ is white Gaussian noise.

The simulated signal and its TFR are presented in Figure 6(a) and (b). In Figure 6(b), the time-varying gear meshing frequency and its harmonics distribute around $1500 \mathrm{~Hz}$, and the IDMM is the second harmonic which conforms with the simulated model. Peak search algorithm is applied to the TFR, and a time-frequency ridge is extracted. The extracted IDMM (blue squares), preset instantaneous meshing frequency (IMF) (red dashed line) and its second harmonic (red solid line) are plotted in Figure 6(c), respectively, in which the IDMM overlaps with the second multiple of the preset IMF. Based on Eq. (4), the IDMM function is calculated as $f_{m d}(t)=108 t+648$, and then the FCF function is obtained by $f_{m d}(t) /(2 \cdot z \cdot c \cdot F)$. Based on Eq. (13), the PF corresponding to the FCF is calculated as $s_{0}(t)=3.75 t^{2}$, and the FP is $45 \mathrm{~Hz}$.

From Figure 6(b), the high frequency resonance band excited by bearing fault also can be easily found, and it distributes from around $4000 \mathrm{~Hz}$ to $6000 \mathrm{~Hz}$. This resonance band has an obvious difference with the gear meshing vibration. Hence, it can be obtained via the band-pass filter algorithm, and the filtering parameters are estimated from Figure 6(b).

Applied the GDT to the envelope signal (Figure 6(d)) with the $s_{0}(t)$, and the spectrum of the demodulated signal is presented in Figure 6(e). It can be seen that a peak whose value is $44.4 \mathrm{~Hz}$ is clearly identified, and

Table 1 Parameters of simulated model

\begin{tabular}{ll}
\hline Parameter & Value \\
\hline Length of the signal $t(\mathrm{~s})$ & 4 \\
Sampling rate $f_{s}(\mathrm{~Hz})$ & 20000 \\
$\mathrm{FCC}$ & 3 \\
Structural damping characteristic $\beta$ & 750 \\
Bearing resonance frequency $w(\mathrm{~Hz})$ & 5000 \\
Number of teeth $z$ & 18 \\
Slippage coefficient $u$ & 0.01 \\
Rotating frequency ratio $c$ & 1.2 \\
\hline
\end{tabular}




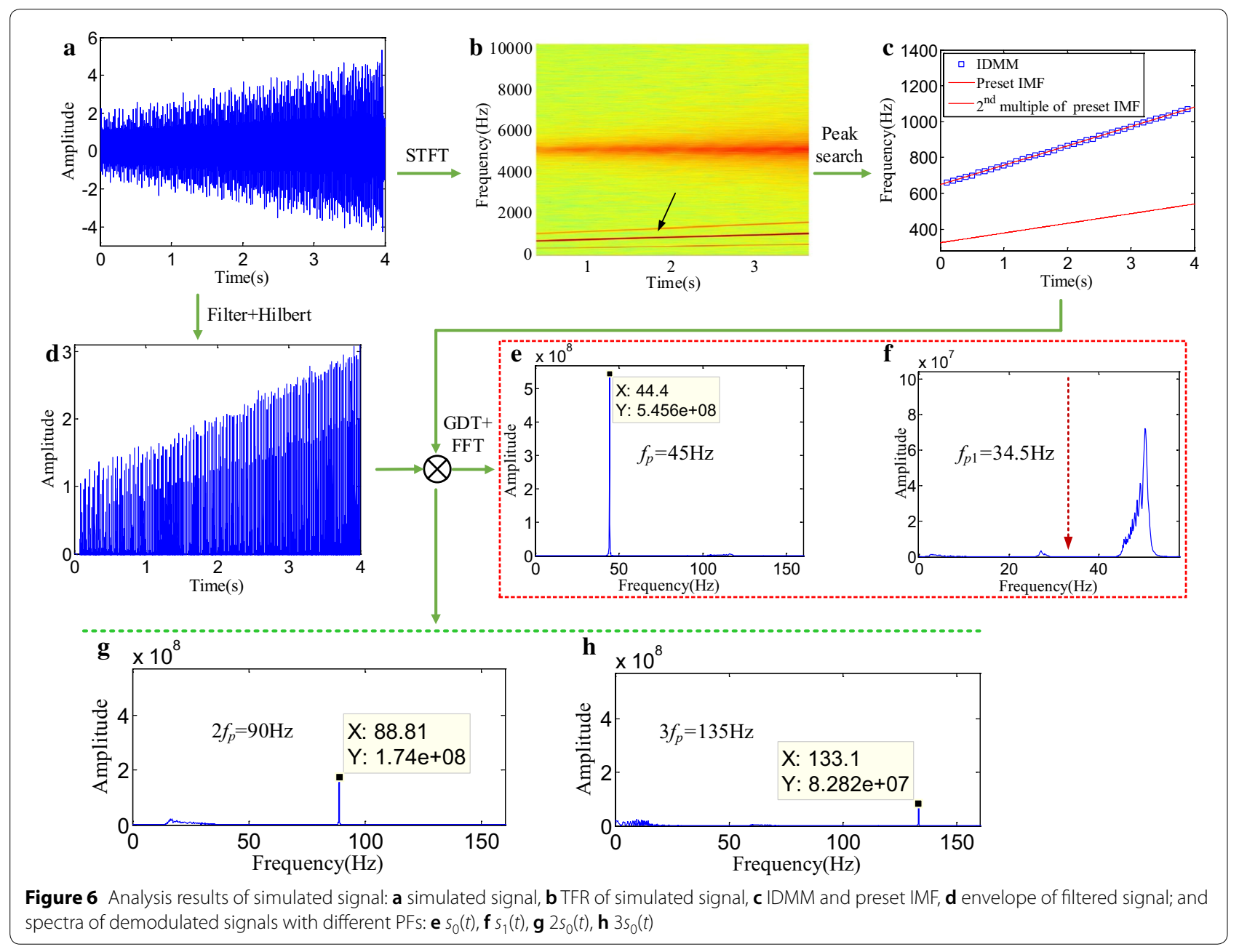

approximately equal to the theoretical FP $f_{p}=45 \mathrm{~Hz}$, which corresponds to the preset fault type. In addition, its harmonics are also located in the corresponding spectra of demodulated signals as shown in Figure 6(g) and (h), respectively. The demodulated signals are calculated by the $2 s_{0}(t)$ and $3 s_{0}(t)$, respectively.

In comparison, an arbitrary FCC (2.3) is selected. A new PF $s_{1}(t)=2.875 t^{2}$ is calculated, and the FP is $34.5 \mathrm{~Hz}$. Based on the new PF, the spectrum is obtained as shown in Figure 6(f), in which there isn't obvious peak corresponding to the $\mathrm{FP} f_{p 1}=34.5 \mathrm{~Hz}$.

The above analysis results indicate that the GDT-based bearing fault diagnosis method is capable to detect bearing fault type under nonstationary conditions and gear noise interferences.

\section{Experimental Verifications}

In this section, the proposed method is further examined using the measured faulty bearing signals. Figure 7 shows the experimental setup. The motor is used to drive

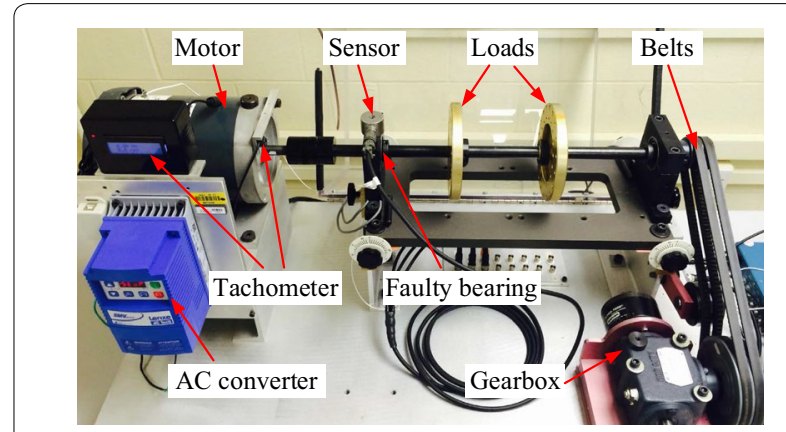

Figure 7 Experimental setup for bearing fault diagnosis

the steel shaft which is supported by two bearings including a faulty one. Two flywheels were installed on the shaft as loads. The shaft rotation speed is controlled by an AC converter, measured by a built-in tachometer. An accelerometer is mounted on the top surface of the faulty bearing housing to measure its vibration signal, and the data 


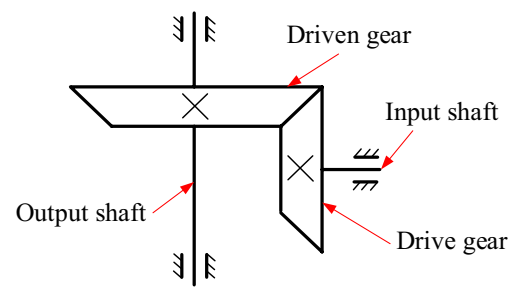

Figure 8 Structure sketch of gearbox

Table 2 Parameters of bearing and gearbox

\begin{tabular}{ll}
\hline Parameter & Value \\
\hline Bearing type & ER-10k \\
Rolling element diameter $d(\mathrm{~mm})$ & 7.94 \\
Pitch diameter $D(\mathrm{~mm})$ & 33.5 \\
Number of rolling elements $n_{b}$ & 8 \\
Contact angle $a$ & 0 \\
FCC of the outer race $F_{o}$ & 3.052 \\
FCC of the inner race $F_{i}$ & 4.95 \\
FCC of the rolling element $F_{b}$ & 1.99 \\
Number of pinion teeth $z_{g}$ & 18 \\
Transmission ratio of belts $i_{b}$ & 2.56 \\
Transmission ratio of gear box $i_{g}$ & 1.5 \\
Serial number of the IDMM $c_{d}$ & 3 \\
\hline
\end{tabular}

acquisition system is mainly consisted of a DAQ card (NI-PCI6259) and NI-DAQmx software. On the right, a single stage bevel gearbox is connected by two belts and two sheaves (the smaller one is fixed on the bearing shaft and the larger one is installed on the gearbox shaft). Structure sketch of the gearbox is shown in Figure 8. The detailed parameters of faulty bearing and gearbox are shown in Table 2.

\subsection{Case 1: Target Bearing with Outer Race Fault}

In this subsection, outer race fault bearing signal is measured from the test rig to verify the proposed algorithm. In this test, the rotational frequency changes from $23.25 \mathrm{~Hz}$ to $31.1 \mathrm{~Hz}$ at first and then goes down to $25.61 \mathrm{~Hz}$ in $4.5 \mathrm{~s}$. The sampling rate is $48000 \mathrm{samples} / \mathrm{s}$.

The measured vibration signal is presented in Figure 9(a). Figure 9(b) and (c) show the TFR of the raw signal and its enlargement of lower band, respectively. In Figure 9(c), a time-varying frequency ridge is dominant, and has same tendency with rotational frequency of the target bearing. The peak search algorithm is applied to the TFR, and the result (blue squares) is plotted in Figure 9(d). For comparison, the IMF (red dashed line) and its third multiple (red solid line) are also plotted in Figure 9(d). The IMF is calculated by the measured rotational frequency, transmission ratio of the belts $i_{b}$ and number of pinion teeth $n_{g}$ (i.e., the $\operatorname{IMF} f_{m}(t)=f(t) / i_{b} \times z_{g}$ ). As shown in Figure 9(d), the IDMM almost coincides with the third multiple of the IMF.

Based on Eq. (4), the IDMM function can be calculated as $f_{m d}=2.5 t^{5}-23 t^{4}+69.8 t^{3}-84 t^{2}+99.4 t+490.4$. Based on Eq. (13), the PF of the IDMM function is obtained by $s_{0}(t)=0.42 t^{6}-4.6 t^{5}+17.45 t^{4}-28 t^{3}+49.7 t^{2}$. Hence, the PFs and FPs used to detect bearing fault are calculated, as shown in Table 3, where $C_{s}=i_{b} \times s_{0}(t) /\left(z_{g} \times c_{d}\right)$, and $C_{f}=490.4 \times i_{b} /\left(z_{g} \times c_{d}\right)$.

It can be seen from Figure 9(b), the high frequency resonance band excited by bearing fault distributes from around $7000 \mathrm{~Hz}$ to $10000 \mathrm{~Hz}$, which has obvious difference with the gear meshing vibration. As a result, the resonance band can be extracted by the band-pass filter with parameters $7000 \mathrm{~Hz}$ and $10000 \mathrm{~Hz}$.

Then, the GDT is applied to the envelope signal with the PFs as shown in Table 3. The spectra of the demodulated signals are presented in Figure 9(f), (g) and (h), respectively. From the figures, only one spectrum obtained by the $s_{o}(t)$ has the obvious peak, whose value is approximately equal to the $f_{p o}$. Furthermore, its harmonics are also detected in the corresponding spectra of the demodulated signals as shown in Figure 9(i) and (j), respectively. The analysis results reveal that the target bearing has an outer race fault.

In order to visualized illustrate the effectiveness of this method, the comparison results between the TFRs of the envelope signal and demodulated signals are presented in Figure 10. The time-varying FCF trend and its harmonics, labeled as (1), (2) and (3) in Figure 10(a) are transformed into lines paralleling to the time axis as shown in Figure 10(b), (c) and (d), respectively.

\subsection{Case 2: Target Bearing with Inner Race Fault}

To verify the proposed algorithm, the measured inner race fault bearing signal is considered. The vibration signal is shown in Figure 11(a), its rotational frequency drops from $30.97 \mathrm{~Hz}$ to $25.12 \mathrm{~Hz}$ in $2 \mathrm{~s}$. The sampling rate is 48000 samples/s. The TFR of the raw signal is presented in Figure 11(b) in which the resonance band excited by the faulty bearing distributes around from $7000 \mathrm{~Hz}$ to $10000 \mathrm{~Hz}$. The partial enlargement of the TFR is depicted in Figure 11(c), which clearly reveals one of the time-varying frequency ridges, and the timefrequency ridge can be extracted using peak search algorithm. The searching result is plotted in Figure 11(d) in which the extracted IDMM (blue squares) overlaps with the third multiple of the IMF (red solid line). The IDMM function is presented as $f_{m d}=-0.89 t^{3}+0.61 t^{2}-$ $58.36 t+651$. Based on Eq. (13), the PF and FP of the above 


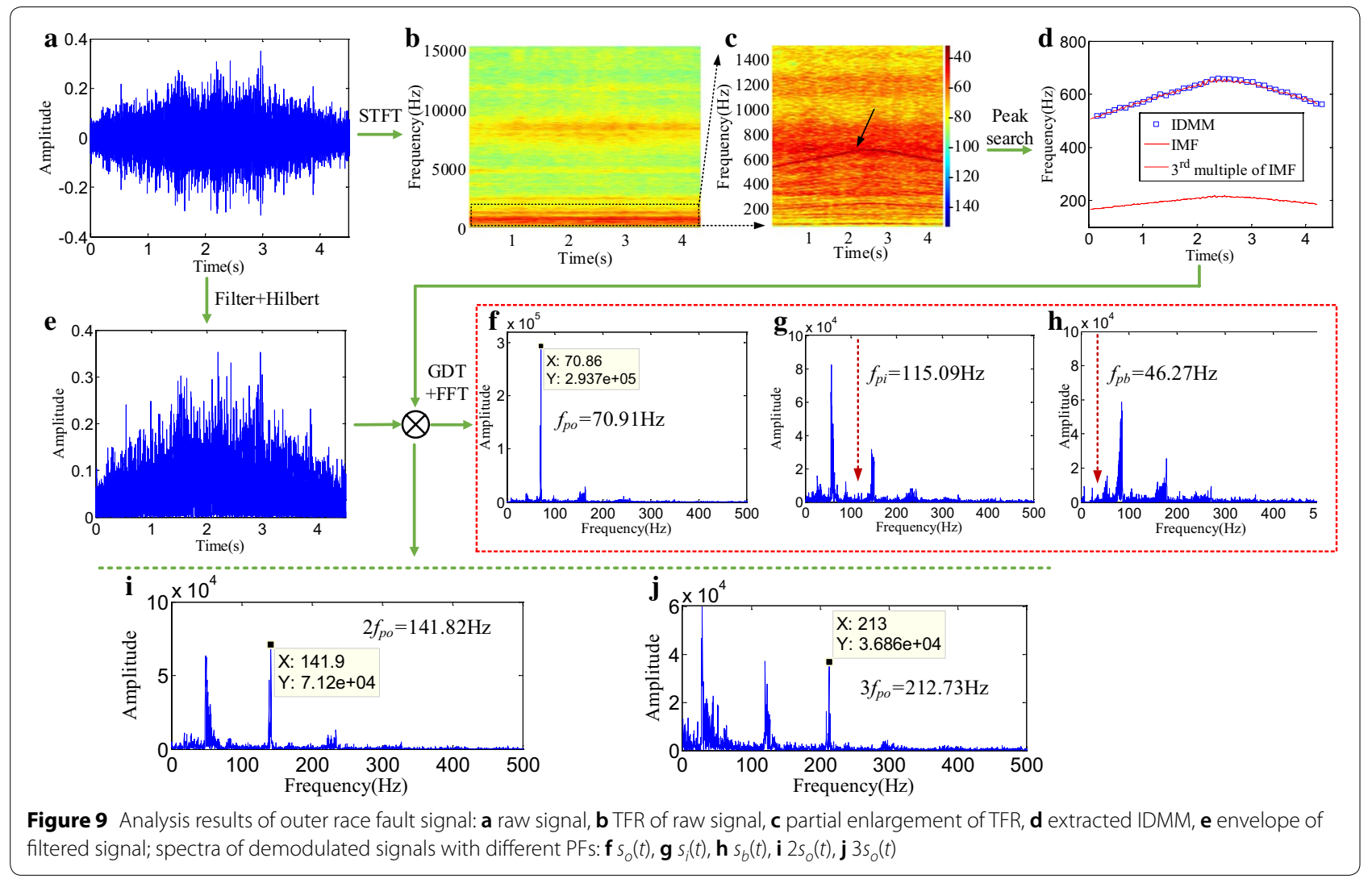

Table 3 PFs and FPs under outer race fault

\begin{tabular}{lll}
\hline Fault type & PF & FP \\
\hline Outer race & $S_{o}(t)=F_{o} \times C_{s}$ & $f_{p o}(t)=F_{o} \times C_{f}=70.91 \mathrm{~Hz}$ \\
Inner race & $S_{i}(t)=F_{i} \times C_{s}$ & $f_{p i}(t)=F_{i} \times C_{f}=115.09 \mathrm{~Hz}$ \\
Ball & $S_{b}(t)=F_{b} \times C_{s}$ & $f_{p b}(t)=F_{b} \times C_{f}=46.27 \mathrm{~Hz}$ \\
\hline
\end{tabular}

function can be calculated as $s_{0}(t)=-0.22 t^{4}+0.2 t^{3}+$ $29.18 t^{2}$ and $f_{p m}=651 \mathrm{~Hz}$.

Based on the FCCs, the PFs corresponding to different fault types are calculated as shown in Table 4, where $C_{s}=i_{b} \times s_{0}(t) /\left(z_{g} \times c_{d}\right)$, and $C_{f}=490.4 \times i_{b} /\left(z_{g} \times c_{d}\right)$.
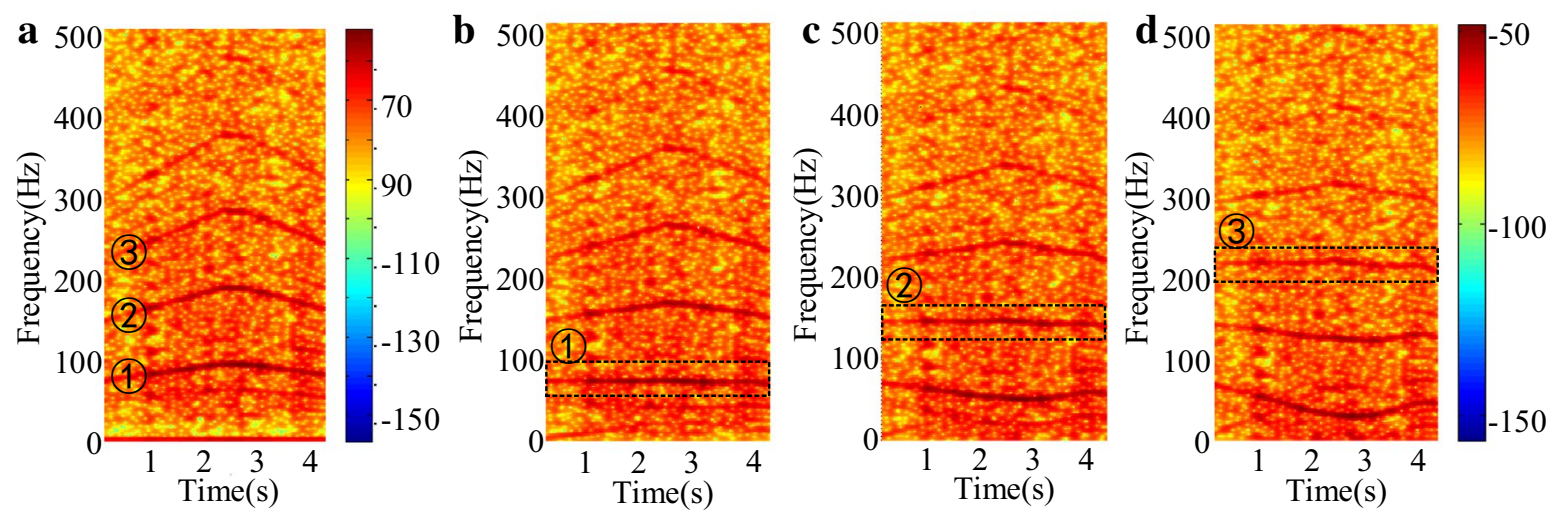

Figure 10 TFR comparison: a TFR of envelope signal; TFR of demodulated using different PFs: $\mathbf{b} s_{0}(t), \mathbf{c} 2 s_{0}(t), \mathbf{d} 3 s_{0}(t)$ 


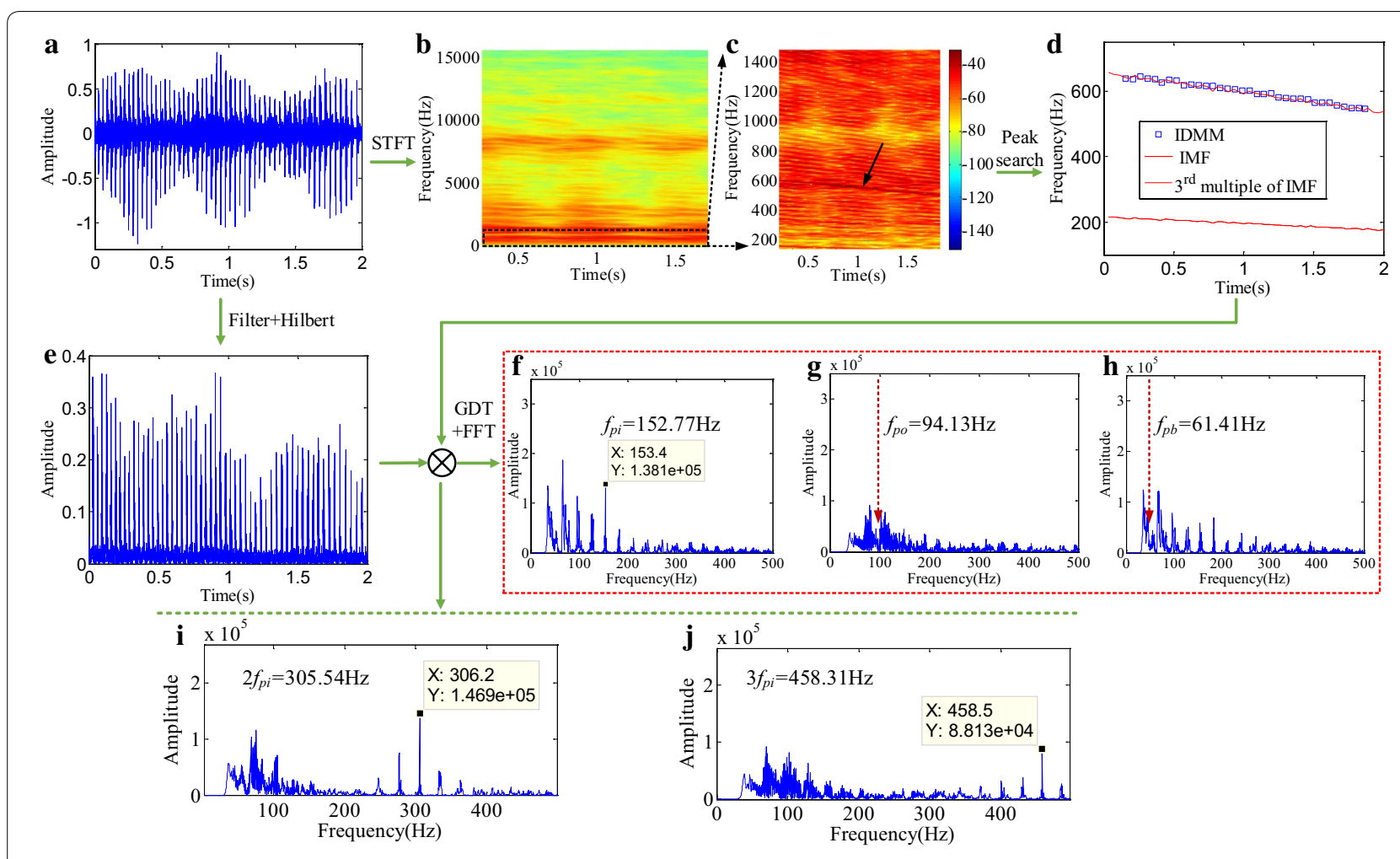

Figure 11 Analysis results of inner race fault signal: a raw signal, b TFR of raw signal, c partial enlargement of TFR, d extracted IDMM, e envelope of filtered signal; Spectra of demodulated signals with different PFs: $\mathbf{f} s_{i}(t), \mathbf{g} s_{0}(t), \mathbf{h} s_{b}(t), \mathbf{i} 2 s_{i}(t), \mathbf{j} 3 s_{i}(t)$

Figure 11(e) shows the envelope of the filtered signal. Figure 11(f), (g) and (h) display the spectra of the demodulated signals obtained with the $s_{i}(t), s_{o}(t)$ and $s_{b}(t)$, respectively. In the spectra, only one spectrum has obvious peak at its corresponding FP $f_{p i}=152.77 \mathrm{~Hz}$. Furthermore, the envelope signal is demodulated with the $2 s_{i}(t)$ and $3 s_{i}(t)$, respectively. The spectra are presented in Figure $11(\mathrm{~g})$ and $(\mathrm{h})$, respectively, which reveal the obvious peak near its corresponding FP $2 f_{p i}(t)$ and $3 f_{p i}(t)$, respectively. This finding confirms the inner race fault.

\section{Conclusions}

1. The IDMM trend is prominent in the TFR of the raw signal based on the gear meshing vibration characteristic, hence it can be extracted for estimating the PFs

Table 4 PFs and FPs under inner race fault

\begin{tabular}{lll}
\hline Fault type & $\mathbf{P F}$ & FP \\
\hline Outer race & $S_{0}(t)=F_{o} \times C_{s}$ & $f_{p o}(t)=F_{o} \times C_{f}=94.13 \mathrm{~Hz}$ \\
Inner race & $S_{i}(t)=F_{i} \times C_{s}$ & $f_{p i}(t)=F_{i} \times C_{f}=152.77 \mathrm{~Hz}$ \\
Ball & $S_{b}(t)=F_{b} \times C_{s}$ & $f_{p b}(t)=F_{b} \times C_{f}=61.41 \mathrm{~Hz}$ \\
\hline
\end{tabular}

and FPs corresponding to different fault types. As a result, the proposed method eliminates the dependence on the tachometer;

2. Based on the high frequency resonance phenomenon, the band-pass filter algorithm can be used to remove the gear meshing vibration without complicated preferences selection and calculation;

3. Due to the AM-FM characteristic, the FCF and its harmonics will appear as time-frequency ridges in the envelope TFR of the resonance signal, and the GDT can be used to transform the time-varying frequency ridges into lines paralleling to the time axis. As a result, the spectrum smearing caused by rotational speed fluctuation is removed without the angular resampling. Furthermore, only the interested time-frequency ridges are mapped, which eliminates the interferences of other spectral lines;

4. The bearing fault type is determined by the calculated PFs and FPs, hence the proposed method is free from capturing the peak corresponding to the rotational frequency information in the envelope spectrum or envelope order spectrum. 


\section{Authors' contributions}

WC was in charge of the whole trial; DZ wrote the manuscript; $J L$ and ZH assisted with sampling and laboratory analyses. All authors read and approved the final manuscript.

\section{Author Details}

1 Department of Mechanical Engineering, Tsinghua University, Beijing 100084 China. ${ }^{2}$ School of Mechanical Electronic and Control Engineering, Beijing Jiaotong University, Beijing 100044, China. ${ }^{3}$ Key Laboratory of Vehicle Advanced Manufacturing, Measuring and Control Technology, Ministry of Education, Beijing 100044, China.

\section{Authors' Information}

Dezun Zhao, born in 1990, is currently a postdoctoral fellow at Department of Mechanical Engineering, Tsinghua University, China. His research interests include fault diagnosis of rotating machinery and signal processing.

Jianyong Li, born in 1962, is currently a professor at School of Mechanical Electronic and Control Engineering, Beijing Jiaotong University, China. His research interests include advanced manufacturing processes and systems.

Weidong Cheng, born in 1967, is a professor at School of Mechanical Electronic and Control Engineering, Beijing Jiaotong University, China. His research interests include fault diagnosis of rotating machinery and signal processing.

Zhiyang He, born in 1991, is currently a PhD candidate at School of Mechanical Electronic and Control Engineering, Beijing Jiaotong University, China.

\section{Competing Interests}

The authors declare that they have no competing interests.

\section{Funding}

Supported by National Natural Science Foundation of China (Grant Nos. 51335006 and 51605244).

\section{Publisher's Note}

Springer Nature remains neutral with regard to jurisdictional claims in published maps and institutional affiliations.

Received: 25 July 2017 Accepted: 16 January 2019

Published online: 01 February 2019

\section{References}

[1] K J Shi, S L Liu, J Chao, et al. Rolling bearing feature frequency extraction using extreme average envelope decomposition. Chinese Journal of Mechanical Engineering, 2016, 29(5): 1029-1036.

[2] HY Cui, Y Y Qiao, Y M Yin, et al. An investigation of rolling bearing early diagnosis based on high-frequency characteristics and self-adaptive wavelet de-noising. Neurocomputing, 2016, 216: 649-656.

[3] Y F Li, M J Zuo, K Feng. Detection of bearing faults using a novel adaptive morphological update lifting wavelet. Chinese Journal of Mechanical Engineering, 2017, 30(6): 1305-1313.

[4] F C Li, M Guang, Y Lin. Wavelet transform-based higher-order statistics for fault diagnosis in rolling element bearings. Journal of Vibration and Control, 2008, 14(11): 1691-1709.

[5] B Murugantham, M A Sanjith, B Krishnakumar, et al. Roller element bearing fault diagnosis using singular spectrum analysis. Mechanical Systems and Signal Processing, 2013, 35(1): 150-166.

[6] Z P Feng, M Liang, F Chu. Recent advances in time frequency analysis methods for machinery fault diagnosis: a review with application examples. Mechanical Systems and Signal Processing, 2013, 38(1): 165-205.

[7] Y Yang, H H Wang, J S Cheng, et al. A fault diagnosis approach for roller bearing based on VPMCD under variable speed condition. Measurement, 2013, 46: 2306-2312.

[8] S L Lu, XX Wang, Q B He, et al. Fault diagnosis of motor bearing with speed fluctuation via angular resampling of transient sound signals. Journal of Sound and Vibration, 2016, 385: 16-32.
[9] P Borghesani, R Ricci, S Chatterton, et al. A new procedure for using envelope analysis for rolling element bearing diagnostics in variable operating conditions. Mechanical Systems and Signal Processing, 2013, 38(1): 23-35.

[10] TY Wang, M Liang, J Y Li, et al. Bearing fault diagnosis under unknown variable speed via gear noise cancellation and rotational order sideband identification. Mechanical Systems and Signal Processing, 2015, 62: 30-53.

[11] D Z Zhao, J Y Li, W D Cheng. Feature extraction of faulty rolling element bearing under variable rotational speed and gear interferences conditions. Shock and Vibration, 2015(3): 1-9.

[12] R B Randall, J Antoni. Rolling element bearing diagnostics-a tutorial. Mechanical Systems and Signal Processing, 2011, 25(2): 485-520.

[13] Y Guo, TW Liu, J Na, et al. Envelope order tracking for fault detection in rolling element bearings. Journal of Sound and Vibration, 2012, 331(25): 5644-5654.

[14] K S Wang, P S Heyns. Application of computed order tracking, VoldKalman filtering and EMD in rotating machine vibration. Mechanical Systems and Signal Processing, 2011, 25(1): 416-430.

[15] Y Wang, G H Xu, Q Zhang, et al. Rotating speed isolation and its application to rolling element bearing fault diagnosis under large speed variation conditions. Journal of Sound and Vibration, 2015, 348: 381-396.

[16] M Zhao, J Lin, X Q Xu, et al. Tacholess envelope order analysis and its application to fault detection of rolling element bearings with varying speeds. Sensors, 2013,13: 10856-10875.

[17] P N Saavedra, C G Rodriguez. Accurate assessment of computed order tracking. Shock and Vibration, 2006, 13(1): 13-32.

[18] W D Cheng, R X Gao, J J Wang, et al. Envelope deformation in computed order tracking and error in order analysis. Mechanical Systems and Signal Processing, 2014, 48(1): 92-102.

[19] S Olhede, A TWalden. A generalized demodulation approach to timefrequency projections for multicomponent signals. Proceedings of the Royal Society of London A: Mathematical, Physical and Engineering Sciences, the Royal Society, 2005, 461(2059): 2159-2179.

[20] Z B Yu, Y K Sun, W D Jin. A novel generalized demodulation approach for multi-component signals. Signal Processing, 2016, 118: 118-202.

[21] J S Cheng, Y Yang, D J Yu. Application of the improved generalized demodulation time-frequency analysis method to multi-component signal decomposition. Signal Processing, 2009, 89(6): 1205-1215.

[22] D Z Zhao, JY Li, W D Cheng, et al. Compound faults detection of rolling element bearing based on the generalized demodulation algorithm under time-varying rotational speed. Journal of Sound and Vibration, 2016, 378:109-123.

[23] Z P Feng, X W Chen, M Liang. Joint envelope and frequency order spectrum analysis based on iterative generalized demodulation for planetary gearbox fault diagnosis under nonstationary conditions. Mechanical Systems and Signal Processing, 2016, 76-77: 242-264.

[24] C Li, V Sanchez, G Zurita, et al. Rolling element bearing defect detection using the generalized synchrosqueezing transform guided by time-frequency ridge enhancement. ISA transactions, 2016, 60: 274-284.

[25] Z P Feng, X W Cheng, TY Wang. Time-varying demodulation analysis for rolling bearing fault diagnosis under variable speed conditions. Journal of Sound and Vibration, 2017, 400: 71-85.

[26] R B Randall, N Sawalhi M Coats. A comparison of methods for separation of deterministic and random signals. The International Journal of Condition Monitoring, 2011, 1(1):11-19.

[27] W Wang. Early detection of gear tooth cracking using the resonance demodulation technique. Mechanical Systems and Signal Processing, 2001, 15(5): 887-903.

[28] TY Wang, F L Chu, Q K Han. Fault diagnosis for wind turbine planetary ring gear via a meshing resonance based filtering algorithm. ISA Transactions, 2017, 67: 173-182.

[29] R Q Yan, R X Gao. Hilbert-Huang transform-based vibration signal analysis for machine health monitoring. IEEE Transactions on Instrumentation and Measurement, 2006, 55(6): 2320-2329.

[30] TY Wang, M Liang, JY Li, et al. Rolling element bearing fault diagnosis via fault characteristic order (FCO) analysis. Mechanical Systems and Signal Processing, 2014, 45: 139-153. 